\title{
Uso de Redes Asociativas Pathfinder para el análisis de redes cognitivas de alumnos: el caso del Sistema Métrico Decimal
}

\author{
Isabel Roldán, Luis Ardila, Ricardo González \\ \& José Luís Carvalho
}

\begin{abstract}
Resumen
En el siguiente artículo se aborda la importancia que tiene el Sistema Métrico Decimal (SMD) en la enseñanza de las matemáticas, y en su aplicación en el resto de las ciencias. El propósito principal es el de estudiar las Redes Cognitivas en el aprendizaje del SMD. La muestra está formada por alumnos del primer curso de Educación Secundaria Obligatoria en el Sistema Educativo español. Para este estudio se empleó un enfoque mixto (cualitativo-cuantitativo) el cual tiene como base, la Teoría de los Conceptos Nucleares (TCN). Se representaron gráficamente las estructuras cognitivas de los alumnos y se analizaron a través de las Redes Asociativas Pathfinder (RAP). Otro de los propósitos del estudio es el de estudiar la forma en la que se produce el aprendizaje, a partir de los cambios en las estructuras cognitivas de los alumnos, así como analizar la estabilidad de dichas estructuras. En términos generales, y a modo de conclusión, podemos decir que la estructura de los estudiantes de $1^{\circ}$ de la ESO es menos compleja que la de su profesora y, a su vez, menos compleja que la estructura de la Red Básica Conceptual (RBC), además de otras conclusiones que se pueden observar en el artículo.
\end{abstract}

sistema métrico decimal; teoría de los conceptos nucleares (TCN); representación del conocimiento; redes asociativas pathfinder (RAP). 


\title{
Use of Pathfinder of Associative Networks for analysis of the cognitive networks of students: the case of Metric Decimal System
}

\begin{abstract}
The Metric Decimal System (MDS) is a considered an important subject either in the teaching of mathematics and in the rest of sciences. The current article is about the MDS, with the purpose of studying the Cognitive Networks in their learning within the students of the first year of Compulsary Secondary Education. Based on the Theory of Nuclear Concepts (TNC) and using a mixed approach (quantitative-qualitative), the cognitive structures of the students, related to the fundamental concepts of MDS, were graphically represented. Then, they were analyzed through Pathfinder Associative Networks (PAN). The main aim of the research was to study how learning occurs from changes in student's cognitive structures and their stability. In general terms we can said that cognitive structure of students a first year of Compulsary Secondary Education with respect to the MDS is a less complex structure than the structure of the teacher and, at the same time, the structure of the basic conceptual network (BCN). It is also appropriate to say that the PAN of the students are different from the red of the teacher and the BCN, among other conclusions that can be seen in the complete article.
\end{abstract}

Keywords: metric system; theory of nuclear concepts; representation of knowledge; pathfinder associative networks (PAN).

\section{Uso das Redes Associativas Pathfinder para a análise das redes cognitivas de alunos: o caso do Sistema Métrico Decimal}

Resumo: O Sistema Métrico Decimal (SMD) é um tema de considerável importância no ensino da matemática e na sua aplicação ao resto das Ciências. Neste artigo, o SMD é abordado tendo como objetivo principal o estudo das Redes Cognitivas na aprendizagem do SMD em estudantes do primeiro ano de Ensino Secundário Obrigatório do sistema educativo espanhol ( $\left.1^{\circ} \mathrm{ESO}\right)$. Tendo por base a Teoria dos Conceitos Nucleares (TCN) e utilizando uma abordagem mista (quantitativa-qualitativa), as estruturas cognitivas dos alunos relacionadas com os conceitos fundamentais do SMD, foram representadas graficamente e analisadas através da técnica de Redes Associativas Pathfinder. (RAP) O objetivo principal deste estudo procurou averiguar, a partir de alterações nas estruturas cognitivas dos estudantes e da estabilidade dessas estruturas, como se produz a aprendizagem. Em geral, é possível concluir que as redes cognitivas dos alunos de $1^{\circ} \mathrm{ESO}$ relacionadas com o SMD apresentam uma estrutura menos complexa do que a estrutura do professor e, por sua vez, do que a estrutura da Rede Conceitual Básica (RCB). Também é apropriado dizer que as redes cognitivas dos alunos é muito diferente da rede de professores e da RCB.

Palavras-chave: sistema métrico decimal; teoria dos conceitos nucleares; representação do conhecimento; redes associativas pathfinder.

\section{Utilisation des réseaux associatifs Pathfinder pour l'analyse des réseaux cognitifs des étudiants: le cas du système métrique décimal}

Résumé: Le Système Métrique Décimal (SMD) est un sujet d'importance considérable dans l'enseignement des mathématiques et son application au reste des sciences. Dans cet article, le SMD est abordé avec l'objectif principal d'étudier lesréseaux cognitifs dans l'apprentissage de SMD chez les étudiants de la première année de l'enseignement secondaire obligatoire du système éducatif espagnol (1er ESO). Sur la base de la Théorie des Concepts Nucléaires (TCN) et en utilisant une approche mixte (quantitative-qualitative), les structures cognitives des étudiants liées aux concepts fondamentaux de SMD ont été graphiquement représentées et analysées par la technique Pathfinder Associative Réseaux. (PAR) L'objectif principal de cette étude était d'étudier, à partir des changements dans les structures cognitives des élèves et la stabilité de ces structures, comment l'apprentissage est produit. En général, il est possible de conclure que les réseaux cognitifs des 1ers étudiants ESO liés au SMD ont une structure moins complexe que la structure de l'enseignant et, à son tour, que la structure du Réseau Conceptuel Basique (RCB). I convient également de dire que les réseaux cognitifs des étudiants sont très différent du réseau de l'enseignant et du RCB.

Mots-clés: système métrique décimal; théorie des concepts nucléaires; représentation de la connaissanc; pathfinder associated networks. 


\section{Introducción}

Es necesario dejar constancia de la importancia que tiene, para el hombre, la necesidad de comparar cantidades, medidas, etc. Esto hace que sea útil el desarrollo de un sistema que nos permita trabajar en el tema planteado.

En nuestro día a día, es habitual realizar mediciones, aunque no seamos conscientes de ello. El mero hecho de establecer la relación entre lo que pesa un alimento y su precio, por ejemplo, es una tarea que se hace casi de forma automática y en dicha tarea utilizamos distintos sistemas de medida.

En esta investigación se pretende dar respuesta a algunas preguntas, como pueden ser: ¿se conoce el tamaño de las unidades estandarizadas? ¿Cómo podemos medir o estimar y qué necesitamos para ello? ¿Dónde, cómo y qué se aprende en la escuela sobre este tema?

La Teoría de los Conceptos nucleares de Casas y Luengo (2004) enmarca nuestra investigación. En esta teoría encontramos una técnica asociada, que recibe el nombre de "Redes Asociativas Pathfinder", a través de las cuales podemos representar la estructura del conocimiento (Luengo, 2013). Nos permite, además, averiguar cómo se produce el aprendizaje de las personas mediante un proceso (no invasivo) de obtención de datos.

\section{Propósito de la investigación e hipótesis}

El propósito principal de nuestra investigación se centra en estudiar las redes cognitivas en el aprendizaje del Sistema Métrico Decimal (SMD), en estudiantes de primer curso de la Enseñanza Secundaria Obligatoria (ESO).

Con la TCN y las Redes Pathfinder se representan las estructuras cognitivas de los estudiantes, sobre los conceptos del SMD, de forma analítica y gráfica, y nos permite obtener información sobre el proceso de aprendizaje en función de todos los cambios que se producen en la estructura cognitiva del alumnado.

Como se ha dicho en el apartado de la introducción, el concepto de Sistema Métrico Decimal se considera muy importante en la enseñanza de las Matemáticas y en su aplicación al resto de las ciencias. Así mismo se considera como un instrumento de transmisión de información utilizado a través de los tiempos.

Una aproximación al problema ya fue abordada por Roldán, Soto, Luengo y Carvalho (2017), y sus resultados provisionales fueron presentados en el congreso CIAIQ17. Este artículo, una vez terminada la investigación, presenta dicho estudio con los resultados y análisis definitivos.

Los objetivos los podemos clasificar en: 


\section{Objetivo general}

El objetivo de esta investigación es realizar un estudio sobre las redes cognitivas del alumnado, y la estabilidad de las mismas, a través de una Unidad Didáctica (UD) sobre el Sistema Métrico Decimal.

\section{Objetivos específicos}

Los objetivos específicos se basan en responder a las siguientes preguntas:

Pregunta 1: ¿Cómo aparece representada la estructura cognitiva de un estudiante de $1^{\circ}$ ESO respecto al Sistema Métrico Decimal?

Pregunta 2: ¿Qué diferencias y similitudes tienen las Redes Asociativas Pathfinder del alumnado comparadas con la Red Básica Conceptual y la Red establecida por la profesora?

Pregunta 3: ¿Los alumnos que tienen un mayor rendimiento tienen una mayor similaridad en sus redes que el resto, en relación a la Red Básica Conceptual?

Pregunta 4: Después de haber pasado un tiempo, ¿Cuál es el "poso cultural" que permanece en el alumno? ¿Las redes cognitivas son estables?

\section{Hipótesis}

Alguna de las hipótesis que planteamos fueron:

Hipótesis 1: Las redes de los alumnos estarán alejadas de la Red Básica Conceptual (RBC).

Hipótesis 2: Cuanto mejor es la calificación del estudiante, más cercana será su red a la RBC.

Hipótesis 3: Los estudiantes categorizados como "buenos" tendrán una red más próxima a la $\mathrm{RBC}$.

\section{Fundamentación teórica}

A lo largo del tiempo, las distintas ciencias han intentado dar una explicación a aquellos factores que influyen en el aprendizaje, así como a los fundamentos cognitivos, pedagógicos y psicológicos que los sustentan, formándose así las diferentes teorías del aprendizaje.

Una de las preocupaciones que presenta un interés mayor para las Didácticas y las Ciencias de la Educación, es el hecho de poder conocer y representar de una forma adecuada como se estructura el conocimiento, así como, la forma de representarlo, construirlo y modificarlo.

Toda la información que vamos almacenando en nuestra cabeza se va organizando y los conceptos que incluimos en ella presentan una significación semántica relacionada. Cuanto mayor sea esa relación, más fácil será el recordar esos conceptos. 
Ausubel, Novak y Hanesian (1978), nos decían que el aprendizaje no era más que crear estructuras de conocimiento que permitieran relacionar los nodos existentes con otros totalmente nuevos, ampliando así el conocimiento.

En relación con lo que Ausubel dice, para que el aprendizaje sea significativo es necesario que los conceptos que existen en la estructura cognitiva se relacionen con la nueva información. Esto facilitará el aprendizaje significativo y permitirá tener una estructura cognitiva clara.

\section{La Teoría de los Conceptos Nucleares (TCN)}

La Teoría de los Conceptos Nucleares (Casas y Luengo, 2004; Luengo, 2013) nos muestra una visión sobre la representación y adquisición del conocimiento. Esta teoría surge en el ámbito de la Tesis Doctoral de Casas (2002), dirigida por Luengo, en el que se estudian las estructuras cognitivas de los alumnos en función de un concepto concreto: el concepto de "ángulo".

En su Tesis, Casas explora el aprendizaje del concepto ángulo y nos dice que está asociada a la dificultad originada por la diferencia entre la definición formal y el proceso cognitivo seguido por el alumnado. Cuando un estudiante construye el concepto ángulo lo hace a partir de la definición, de la imagen mental y de los procesos relacionados con su experiencia educativa.

Esta teoría emerge con una intención clara: responder a un problema fundamentado en la Teoría del Aprendizaje de Ausubel.

Esta teoría surge con la intención de responder a un problema que se fundamenta en la teoría del aprendizaje significativo de Ausubel. Para sus autores, la adquisición general del conocimiento y su almacenamiento en una estructura cognitiva sigue un proceso análogo al del conocimiento del medio físico que nos rodea. Para explicarlo nos indican que los conceptos no se aprenden de forma aislada, sino que se van asociando a otros en forma de estructura, y estas a su vez van formando redes.

A su vez, se pudo observar que a medida que pasa el tiempo, los alumnos agrupan su aprendizaje en torno a menos conceptos, aunque estos se encuentran enlazados. Sus redes adquieren cada vez más coherencia y a medida que maduran, su estructura cognitiva va englobando los conceptos parciales (Bizarro et al, 2015).

Los autores llaman conceptos nucleares a aquellos conceptos en torno a los cuales, y de forma mayoritaria, se organizan las redes de los alumnos. No son necesariamente los más generales o los más abstractos sino los que a ellos les resultan más significativos (Contreras et al, 2016).

Considerando que la adquisición del conocimiento sigue un paradigma jerárquico, una estructura cognitiva se vuelve más compleja con el aumento del número de conceptos, pero Casas y Luengo (2004), a la luz de los resultados de su investigación, 
definen los "senderos de mínimo coste" cuando dada una situación que implica utilizar aprendizajes ya adquiridos y almacenados en la estructura compleja el alumno recorre aquellas rutas que dependiendo de los propósitos de cada acaso les resultan más rápidas y simples. Así el alumno resuelve eficazmente los problemas, recorre caminos poco usuales y aplica su creatividad (Roldan, Soto, Luengo y Carvalho, 2017).

\section{Representación del Conocimiento. Redes Asociativas Pathfinder (RAP)}

Según Schvaneveldt (1989), las redes no son más que representaciones en las que los conceptos se reflejan como nodos que se relacionan unos con otros, siendo sus relaciones una serie de segmentos, de longitudes variables, que dependen del peso o fuerza de su proximidad semántica.

La forma de crear estas redes es partir de una serie de conceptos los cuales el sujeto evalúa y establece la proximidad que existe entre ellos. Para hacerlo se utiliza el software GOLUCA (Godinho, Luengo y Casas, 2011), creado por el grupo de investigación Ciberdidact de la Universidad de Extremadura. Lo que hace este software es presentar, de forma aleatoria, todos los pares posibles de conceptos (previamente establecidos), para que el sujeto asigne el nivel de relación que considere oportuno.

Los datos que nos ofrece GOLUCA nos permite calcular una matriz de correlaciones, la cual nos representa los "pesos" de los enlaces entre los conceptos. A partir de esa matriz y utilizando los algoritmos de Kamada y Kawai ofrece una representación gráfica de la que se obtiene la red resultante usando el algoritmo Spring Embedding.

Las matrices obtenidas se tratan mediante técnicas estadísticas (análisis de componentes principales, análisis de Clúster, escalamiento multidimensional o RAP) y transforman los datos de interrelación entre conceptos en distancias entre puntos en un espacio de dimensiones mínimas, de tal manera que se obtiene una representación espacial o se determina la estructura subyacente de los datos.

Lo que las RAP aportan es el poder crear representaciones, en forma de redes, de la estructura cognitiva de los sujetos, mediante una serie de datos empíricos y generados de forma automática (Carvalho, 2011).

\section{La enseñanza aprendizaje del SMD y la estimación}

Como hemos explicado a lo largo del estudio, la necesidad de comparar, hace que sea necesaria la aparición de la medida. Estas comparaciones, comienzan tomando como referencia, las partes del propio cuerpo (braza, pie...), pero con el paso del tiempo, el hecho de que las diferencias entre los distintos sistemas de medida sean tan 
grandes, hace que sea necesaria la unificación de todos ellos en un único sistema con valor universal (Roldan, Soto, Luengo y Carvalho, 2017).

La aparición del SMD fue un intento de superación de los inconvenientes que tenían los sistemas anteriores, por lo que suponía de dificultad en las transacciones comerciales internacionales que obligaban a unas operaciones de equivalencias de gran complejidad.

Picado, Rico y Gómez (2013) nos explican aquellas destrezas previas que conseguirían facilitar la realización de las estimaciones razonables en medida, además de facilitar las estrategias que se pueden utilizar en aquellos procesos de estimación. Estas destrezas previas incluyen: la interiorización de las unidades de medida, el referente, las técnicas indirectas, las estrategias de comparación, y estrategias de descomposición y recomposición.

Los contenidos sobre estimación en medida se desarrollan en los cursos de $1^{\circ}$ y $2^{\circ}$ de la ESO. En el primer curso los contenidos son: Estimación y cálculo de perímetros de figuras. Estimación y cálculo de áreas mediante fórmulas, triangulación y cuadriculación". En el segundo curso, en el Bloque 4. Geometría: Volúmenes de cuerpos geométricos. Resolución de problemas que implican la estimación y el cálculo de longitudes, superficies y volúmenes"

\section{Diseño de la investigación}

Como hemos explicado anteriormente, esta investigación se encuentra bajo el abrigo de las investigaciones del grupo de Investigación Ciberdidact, de la UEx, en el marco de la TCN, pretendiendo conocer las estructuras cognitivas de los alumnos de $1^{\circ}$ ESO en relación al tema del SMD.

Esta investigación entra dentro de los métodos mixtos, dado que requiere de un análisis cuantitativo como cualitativo. El método de recogida de datos fue la "encuesta", aunque en este caso, en vez de usar una encuesta tradicional, se utilizó el software GOLUCA, el cual nos permitiría obtener las redes de los alumnos.

Este software se utilizó en tres momentos diferentes: antes de la realización de la Unidad Didáctica, tras su uso y una vez transcurridos tres meses. El diseño del trabajo de campo se puede ver en la siguiente figura:

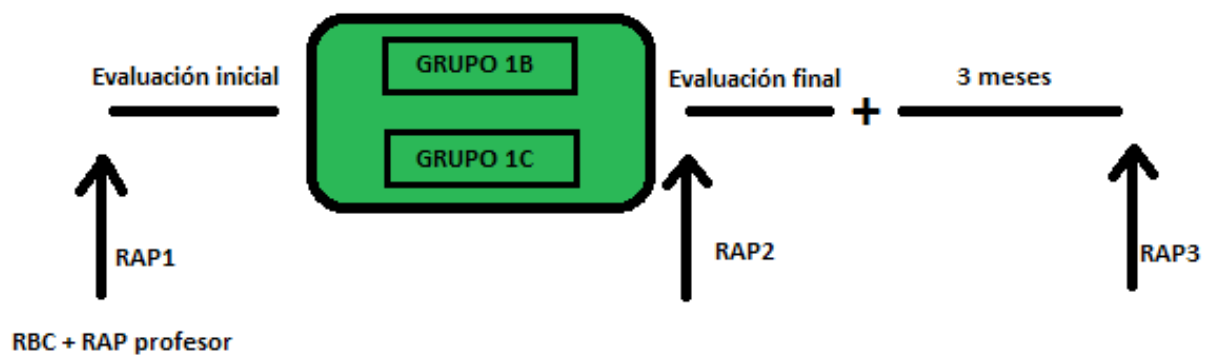

Figura 1. Diseño de la investigación 
A continuación, explicaremos, de forma más detallada el desarrollo de la Figura 1:

En primer lugar, nuestra investigación se inició con la selección de los conceptos más importantes para el análisis de las RAP. Para ello, entrevistamos a los profesores y revisamos libros de texto. Aquellos conceptos más repetidos se emplearían para la elaboración de la RBC.

Una vez definidos los conceptos, pasamos a la realización de una evaluación inicial para evaluar los contenidos que los alumnos tenían interiorizados de su etapa en Primaria. Tras elaborar la RBC analizamos las Redes Conceptuales de los alumnos (RAP1) y la RAP del profesor.

Posteriormente, se impartió la Unidad Didáctica sobre el SMD a los alumnos, en la que se evaluaron los conocimientos adquiridos por los alumnos mediante la realización de una evaluación final que nos permitirá clasificar a los alumnos en función de sus notas. Tras ello, se analizarán las Redes de nuevo (RAP2).

Tres meses después se volvieron a analizar las RAP (RAP3) para comprobar la estabilidad de las Redes de los alumnos con el paso del tiempo para así poder estudiar el "poso cultural" que mantienen sobre el tema del SMD.

La muestra de participantes en la investigación estaba constituida por alumnos de dos grupos del $1^{\circ}$ curso de la ESO del I.E.S "Lacimurga Constantia lulia" de Navalvillar de Pela de la provincia de Badajoz. Los grupos fueron: $1^{\circ} \mathrm{B}$ formado por 25 alumnos y el grupo $1^{\circ} \mathrm{C}$ por 22 alumnos.

\begin{tabular}{|cc|cc|cc|}
\hline NÚMERO & INICIALES & NÚMERO & INICIALES & NÚMERO & INICIALES \\
\hline 1 & CAR & 10 & DFT & 18 & PLGP \\
2 & MAE & 11 & IFC & 19 & DGR \\
3 & MAG & 12 & IGP & 20 & NHM \\
4 & ECS & 13 & MGR & 21 & FMR \\
5 & OCR & 14 & AGC & 22 & MMH \\
6 & MCP & 15 & NGS & 23 & VMM \\
7 & ACU & 16 & DGA & 24 & DMM \\
8 & NFC & 17 & MGL & 25 & AVG \\
9 & JAFM & & & & \\
\hline
\end{tabular}

Figura 2. Alumnos del grupo B 


\begin{tabular}{|cc|cc|cc|}
\hline NÚMERO & INICIALES & NÚMERO & INICIALES & NÚMERO & INICIALES \\
\hline 1 & PAG & 9 & SMB & 16 & ADRP \\
2 & MCB & 10 & IMS & 17 & RRR \\
3 & JCC & 11 & SPS & 18 & JARB \\
4 & MCA & 12 & SPC & 19 & TRM \\
5 & EDA & 13 & IPC & 20 & VSS \\
6 & GGH & 14 & JARD & 21 & AVR \\
7 & SHR & 15 & JARF & 22 & AZG \\
8 & RHS & & & & \\
\hline
\end{tabular}

Figura 3. Alumnos del grupo C

\section{Diseño de la unidad didáctica y selección de conceptos}

La Unidad Didáctica (UD) sobre el SMD propuesta en esta investigación, contribuye a la adquisición de las Competencias Básicas y pretende conseguir unos objetivos ligados a los criterios de evaluación de la misma unidad. Dichos contenidos están sacados del currículum y de los libros de texto analizados ad hoc.

Como podemos ver en la figura 4, los objetivos de nuestra UD son los siguientes

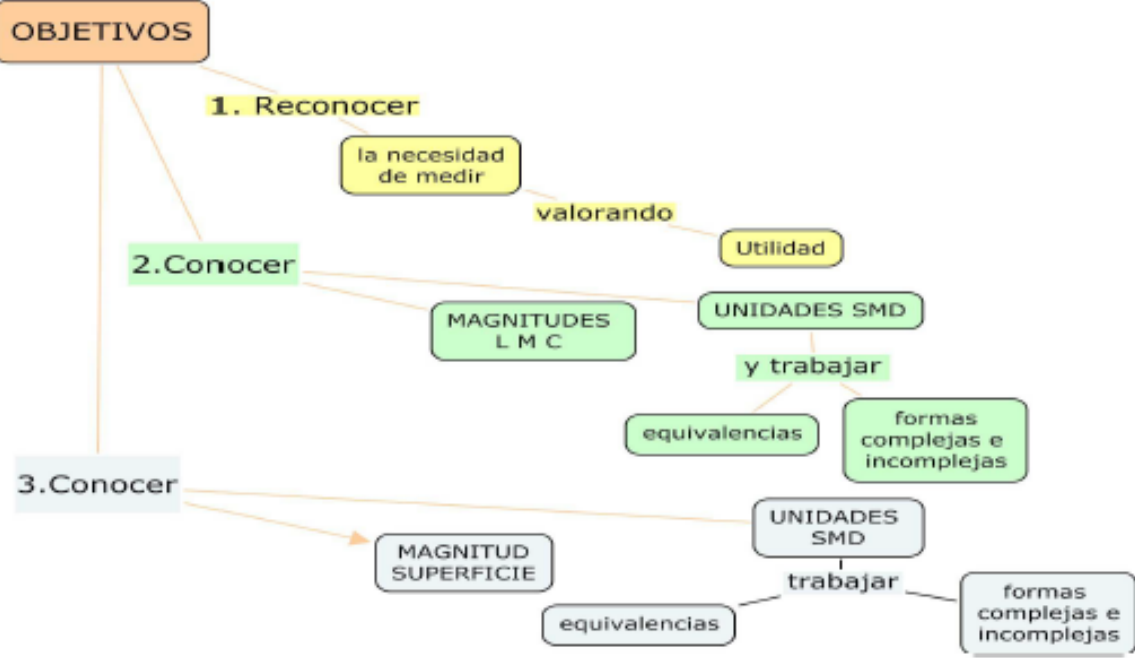

Figura 4. Esquema de los objetivos de la UD 
Para la evaluación final de la UD, tendremos en cuenta los siguientes criterios de calificación:

Tabla 1. Criterios de calificación de la evaluación final

\begin{tabular}{|c|c|c|}
\hline CALIFICACIÓN & INSTRUMENTO & PORCENTAJE \\
\hline $\begin{array}{c}\text { Calificación de } \\
\text { conceptos }\end{array}$ & $\begin{array}{c}\text { Preguntas orales } \\
\text { Constatación de su adquisición a través de su aplicación } \\
\text { práctica }\end{array}$ & $40 \%$ \\
\hline $\begin{array}{c}\text { Calificación de } \\
\text { procedimientos }\end{array}$ & $\begin{array}{c}\text { Ejercicios resueltos en la pizarra } \\
\text { Trabajos de aplicación (individuales y en grupo) } \\
\text { Cuaderno de clase }\end{array}$ & $30 \%$ \\
\hline $\begin{array}{c}\text { Calificación de } \\
\text { las actitudes }\end{array}$ & $\begin{array}{c}\text { Observación diaria en clase } \\
\text { Trabajo en casa }\end{array}$ & $30 \%$ \\
\hline
\end{tabular}

En cuanto a la selección de los conceptos, se realizaron revisiones bibliográficas sobre el tema y se entrevistaron a los docentes. También se analizaron los libros de texto de $1^{\circ}$ ESO utilizados en la Comunidad Autónoma de Extremadura. De eso se obtuvo una lista inicial sobre los conceptos relacionados con el SMD. Posteriormente, se seleccionaron los conceptos más relevantes, tomando como criterio de selección la frecuencia de aparición en los textos, por lo tanto, los términos seleccionados serán aquellos que más aparecen en la literatura. Los conceptos elegidos para el estudio fueron: Magnitud; Unidad; Medir; Instrumentos de medida; SMD; Múltiplos y Submúltiplos de 10; Aproximar; Contar.

\section{Red Básica Conceptual (RBC) y red de la profesora}

La RBC se puede definir como "la red de la ciencia", es decir, es una red formada por aquellos conceptos que se consideran elementales sobre un tema concreto. En este caso, la RBC es la representación gráfica de los conceptos del SMD que la ciencia considera de mayor importancia (Roldán, 2013).

Esta red nos permite determinar el grado de similitud de las redes de los alumnos, tanto antes de realizar nuestra experiencia, como después. Esta red (al igual que el resto) ha sido creada por el Software GOLUCA, basándonos en los criterios que fueron aplicados en los estudios de Hidalgo (2007) y Arias (2008). 


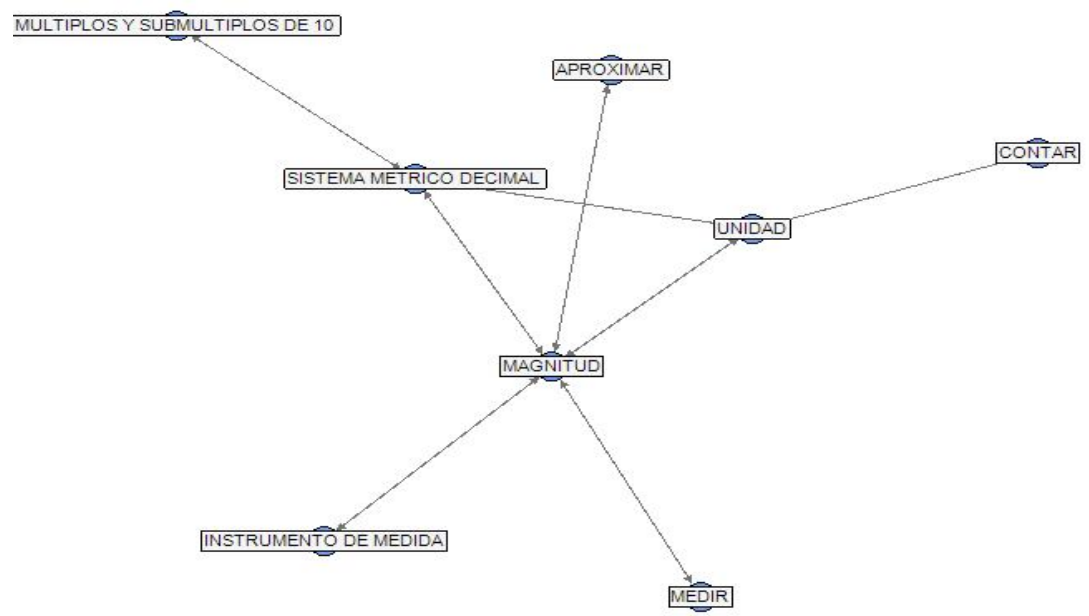

Figura 5. Red Básica Conceptual (RBC)

Con respecto a la profesora, tras evaluar sus relaciones entre los conceptos más importantes sobre el tema (a través del software GOLUCA), obtuvimos la siguiente red:

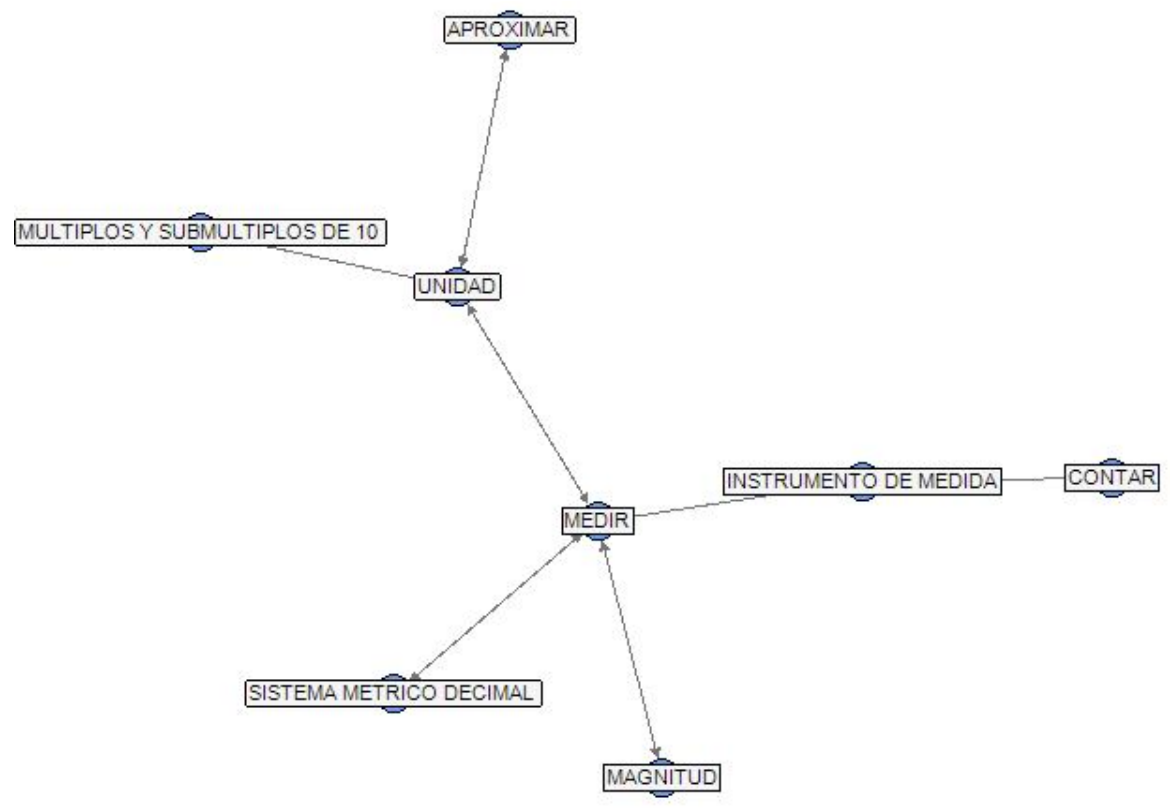

Figura 6. Red de la profesora 


\section{Procedimientos de análisis de los datos}

Para analizar nuestros datos, se llevaron a cabo varios procedimientos interrelacionados. Por un lado, se analizaron los resultados obtenidos tras evaluar la UD y las calificaciones de los alumnos y por el otro, las redes de esos alumnos y establecer una relación con la RBC y la red de la profesora.

Las redes se evaluarán a partir de tres indicadores:

- Nodos nucleares o múltiples: conceptos que tienen más de dos enlaces en la estructura cognitiva del alumno. Representan conceptos que sirven como anclajes a la estructura cognitiva de tal forma que cuanto mayor es el número de nodos nucleares más son las posibilidades de anclar los conceptos nuevos con los conceptos previos.

- Índice de coherencia: Mide la consistencia de las relaciones de conceptos y se evalúa el grado de atención y concentración del alumno el momento de la prueba. Este índice oscila entre -1 y 1, de tal forma que cuanto mayor es el valor mayor será la coherencia de la red.

- Índice de complejidad estructural: Mide la complejidad de la red. Su valor oscila entre 0 a 1, cuanto más próxima a 0 la red será más simple o menos compleja y cuanto más próxima a 1 la red será más compleja.

- Índice de similaridad entre dos redes: Permite determinar la semejanza o acercamiento entre las redes. Sus valores van desde 0 a 1 de tal forma que dos redes serán idénticas si tienen un índice de similitud 1 y dos redes no tienen ninguna similitud si el índice de similaridad es 0 . En esta investigación se estudia la similaridad de las RAP de los alumnos con la RBC y con la red de la profesora.

\section{Análisis y discusión de los resultados}

\section{Análisis de las calificaciones de los alumnos}

Para comenzar con el análisis de nuestro estudio, comenzamos por obtener las calificaciones que los alumnos obtuvieron en la evaluación final que se les realizo. Esto nos permitió clasificarlos en función de los resultados obtenidos, quedando la siguiente distribución: Alumnos "buenos", entre los que se incluyen aquellos que sacaron una puntuación mayor que 8, alumnos "normales", con una puntuación entre 5 y 8 y alumnos que "presentan dificultades", que eran los alumnos que no aprobaron el examen.

Con respecto a las calificaciones obtenidas tras la evaluación de la UD, obtuvimos los siguientes resultados: 


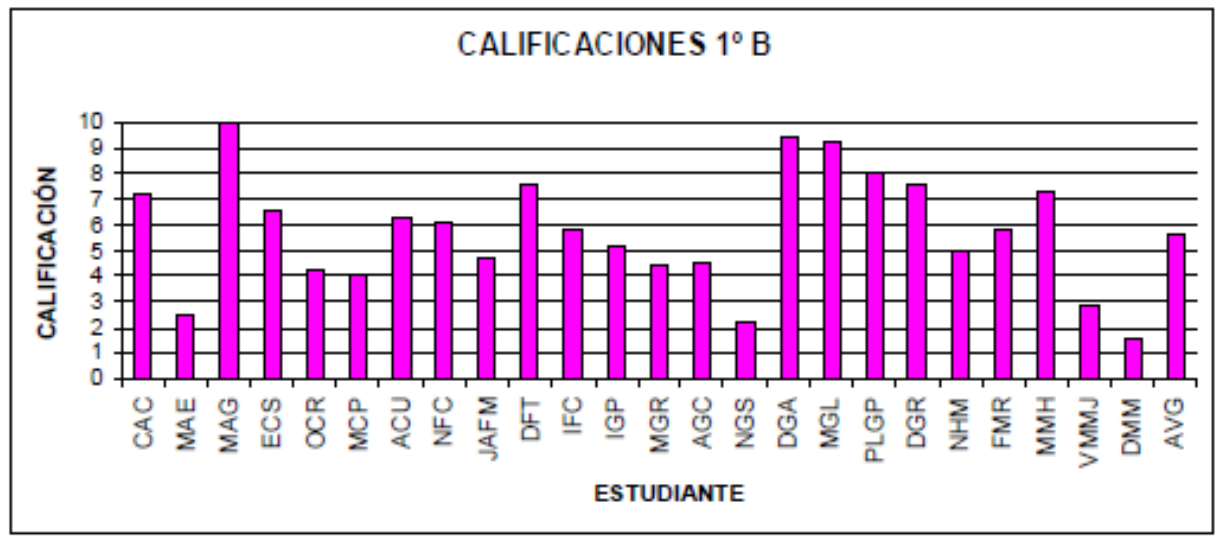

Figura 7. Calificaciones $1^{\circ} \mathrm{B}$

En estos datos se observa que de los 25 estudiantes que forman el grupo B, 16 de ellos (64\%) superan la evaluación final y los 9 restantes (36\%) presentan dificultades. Además, también se puede observar que de los 16 estudiantes que superan la prueba, a 4 estudiantes (16\%) se les considera "buenos" por tener una calificación superior a 8 y a 12 estudiantes (48\%) se les considera "normales" por haber obtenido una calificación entre 5 y 8 . Estos datos se representan gráficamente en la figura 8.

\section{CLASIFICACIÓN DE ESTUDIANTES DE $1^{\circ} \mathrm{B}$}
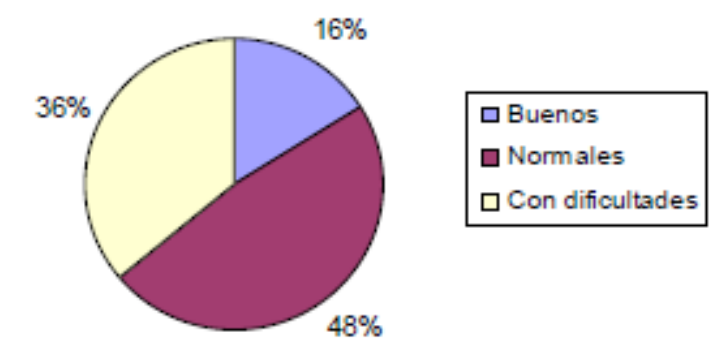

Figura 8. Clasificación de los alumnos según sus notas 
Con respecto a los alumnos del grupo C, obtuvimos los siguientes resultados:

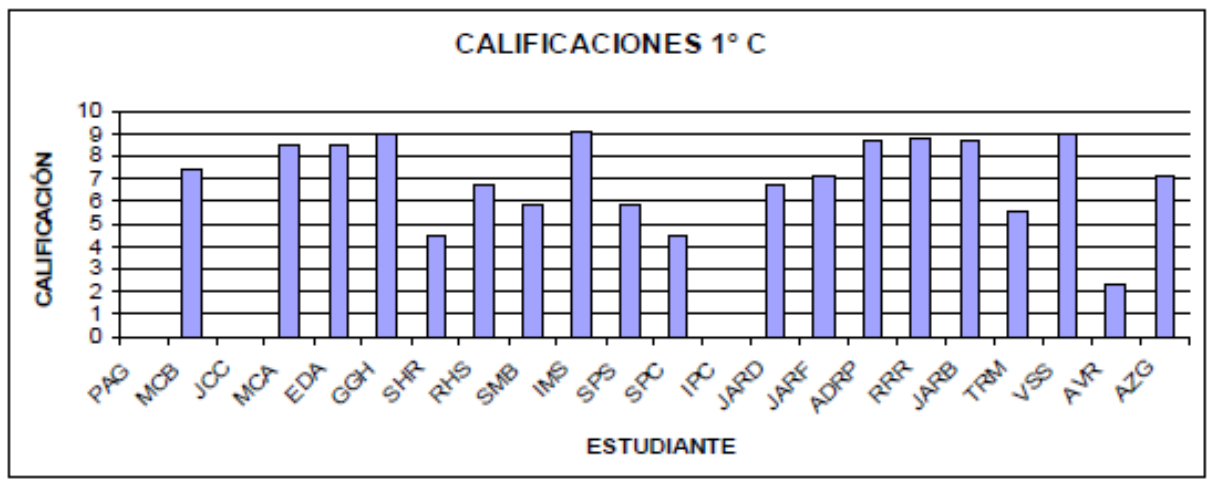

Figura 9. Calificaciones $1^{\circ} \mathrm{C}$

De los 16 estudiantes que superan la prueba, a 8 estudiantes (36\%) se les considera "buenos" por tener una calificación superior a 8 y a 8 estudiantes (36\%) se les considera "normales" por haber obtenido una calificación entre 5 y 8, los 6 alumnos restantes (27\%) son alumnos que "presentan dificultades".

\section{CLASIFICACIÓN DE ESTUDIANTES DE $1{ }^{\circ} \mathrm{C}$}

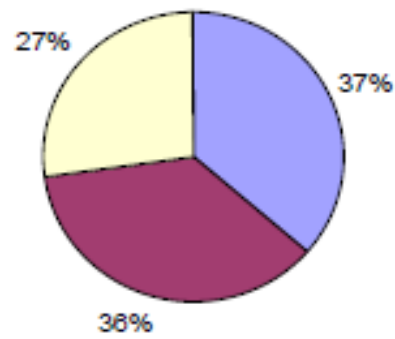

$\square$ Buenos

$\square$ Normales

$\square$ Con dificultades

Figura 10. Clasificación de los alumnos según sus notas 


\section{Análisis de los indicadores de evaluación de las redes}

Tras obtener las calificaciones de los alumnos y clasificarlos por grupos, pasamos al análisis de los distintos indicadores que nos permiten evaluar las redes.

\section{Índice de coherencia de las redes medias}

Las redes medias son calculadas a partir de los valores de los pesos entre los conceptos de dos o más matrices. Como su nombre indica, una red media resulta de la media de los valores entre cada par de conceptos y puede ser calculada y representada mediante Redes Asociativas Pathfinder, representando, en este caso, al grupo en su conjunto.

Al analizar la coherencia de las redes en función del grupo al que pertenecen los alumnos $\left(1^{\circ} \mathrm{B} \circ 1^{\circ} \mathrm{C}\right)$ obtuvimos la siguiente tabla:

Tabla 2. Clasificación Índice de Coherencia de Redes Medias de Grupos B y C

\begin{tabular}{|c|c|c|c|}
\hline & $\begin{array}{c}\text { Indice de } \\
\text { Coherencia } \\
\text { RAP-1 }\end{array}$ & $\begin{array}{c}\text { Indice de } \\
\text { Coherencia } \\
\text { RAP-2 }\end{array}$ & $\begin{array}{c}\text { Indice de } \\
\text { Coherencia } \\
\text { RAP-3 }\end{array}$ \\
\hline Grupo1B 12 ESO & 0,78389212 & 0,69823901 & 0,17058772 \\
Grupo1C 12 ESO & 0,3875904 & $-0,16352454$ & 0,27137419 \\
\hline
\end{tabular}

Como se puede observar en la tabla 2, la coherencia de las redes es diferente para los dos grupos, siendo en ambos casos, las RAP-1 (procedentes del momento anterior a la impartición de la UD) las que tienen valores superiores, seguidas de las RAP-2 y de las RAP-3. Este resultado indica que las redes iniciales son las más coherentes, o sea, que la concentración de los estudiantes a la hora de realizar el Test de Goluca es máxima en el momento inicial, y después va disminuyendo con el transcurso de la investigación.

Ahora bien, si clasificamos a los alumnos en función de las notas obtenidas, obtenemos otros resultados que pueden resultar interesantes. Como podemos observar en la figura 11, los alumnos presentan un índice de coherencia variable en el tiempo. Las RAP 2 y 3 nos muestran como los alumnos que sacaron mejores notas (los "buenos") tienen una coherencia superior que las del resto, con lo que podemos decir que aumenta con el tiempo (va mejorando su atención e interés sobre los conceptos del SMD). 

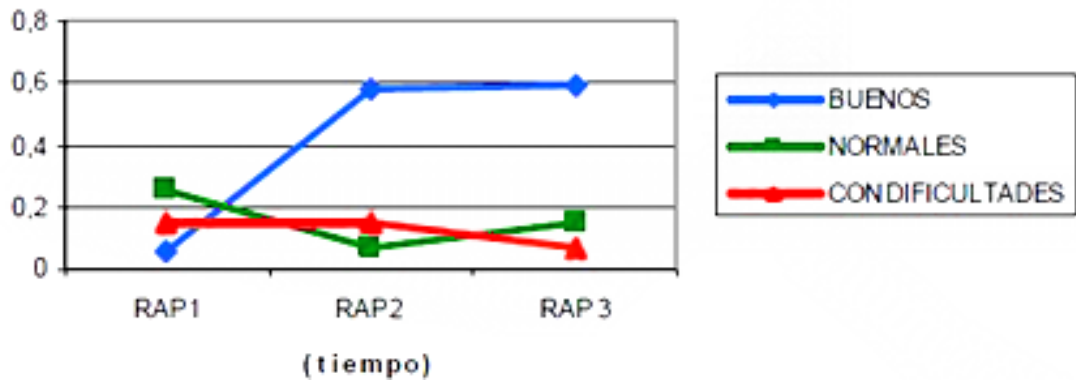

Figura 11. Índice de coherencia para grupos en función de la calificación

La siguiente tabla nos permite ver de forma más exacta estos resultados:

Tabla 3. Clasificación Índice de Coherencia de Redes Medias en función a la calificación

\begin{tabular}{|l|r|c|c|}
\hline & $\begin{array}{c}\text { Indice de } \\
\text { Coherencia } \\
\text { RAP-1 }\end{array}$ & $\begin{array}{c}\text { Indice de } \\
\text { Coherencia } \\
\text { RAP-2 }\end{array}$ & $\begin{array}{c}\text { Indice de } \\
\text { Coherencia } \\
\text { RAP-3 }\end{array}$ \\
\hline BUENOS & 0,06218985 & 0,57425083 & 0,59171885 \\
NORMALES & 0,25 & 0,07142857 & 0,15384615 \\
\hline
\end{tabular}

Índice de Complejidad Estructural de las redes medias

Con respecto a este índice, analizaremos de la misma forma que hicimos en el apartado anterior, primero clasificándolos en función del grupo de clase al que pertenecen y posteriormente en función a sus notas. 
Tabla 4. Clasificación Índice de Complejidad de Redes Medias de Grupos B y C, RBC y red de la profesora

\begin{tabular}{|c|c|c|c|}
\hline & I.C.E. RAP-1 & I.C.E. RAP-2 & I.C.E. RAP-3 \\
& & & \\
\hline Grupo1B 10 ESO & 6,69642857 & 7,8125 & 6,69642857 \\
Grupo1C 19 ESO & 6,69642857 & 6,69642857 & 1,67410714 \\
RBC & 21,0459184 & & \\
Red de la profesora & 7,8125 & & \\
\hline
\end{tabular}

En la tabla anterior (tabla 4) podemos observar que los valores del índice de complejidad de las redes medias de los estudiantes de $1^{\circ} \mathrm{B}$ y $1^{\circ} \mathrm{C}$ están, en general, en torno al mismo valor, lo que corrobora que las redes cognitivas de los estudiantes de $1^{\circ}$ ESO tienen aproximadamente la misma complejidad. Además, dado que los valores del índice de complejidad de la red media de los estudiantes son inferiores a los de la red de la profesora y a su vez a los de la RBC, se deduce que las redes cognitivas de los estudiantes son más simples que la red de la profesora, y que la RBC.

En la tabla 5 y en la figura 12, se recogen los datos obtenidos para el índice de complejidad estructural (I.C.E.) de las Redes Medias de los grupos obtenidos según la calificación en la evaluación de la UD:

Tabla 5. Clasificación Índice de Complejidad de Redes Medias de los alumnos según sus notas

\begin{tabular}{|c|c|c|c|}
\hline & I.C.E. RAP1 & I.C.E. RAP2 & I.C.E. RAP3 \\
\hline BUENOS & 8,92857143 & 7,8125 & 2,23214286 \\
NORMALES & 1,67410714 & 2,23214286 & 6,69642857 \\
CON DIFICULTADES & 6,69642857 & 6,69642857 & 6,69642857 \\
\hline
\end{tabular}




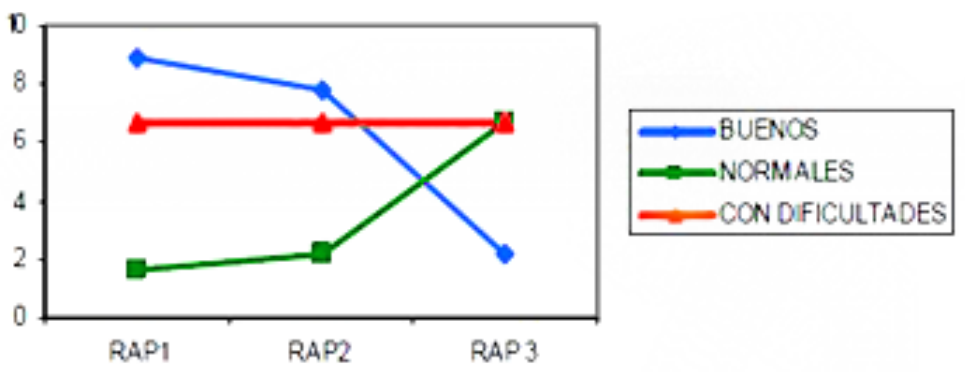

(tiempo)

Figura 12. Índice de complejidad para grupos en función de la calificación

Como podemos observar en la tabla 5 y en la figura 12, los alumnos "buenos" presentan unas redes con una complejidad mayor que la del resto de sus compañeros, tanto al principio como al final de la experiencia con la Unidad Didáctica. Sin embargo, en el transcurso del tiempo, cuando analizamos las RAP3, observamos que esa complejidad ha disminuido, permitiéndonos hacer mención a "los senderos del mínimo coste", expuestos por Casas y Luengo (2004), los cuales nos explican que, con el tiempo, el individuo se olvida de aquellos conceptos que no les resultan importantes, centrándose en los que tenía más interiorizados.

Por otro lado, podemos observar las redes de los alumnos que "presentan dificultades", los cuales mantienen la complejidad de sus redes a lo largo del tiempo, algo que se justifica con las dificultades que presentan a la hora de aprender conceptos nuevos.

\section{Conceptos nucleares de las redes medias}

Los Conceptos Nucleares para las redes medias de los grupos $1^{\circ} \mathrm{B}$ y $1^{\circ} \mathrm{C}$ se reflejan en la siguiente tabla:

Tabla 6. Clasificación de los Conceptos Nucleares de las Redes Medias de los Grupos B y C, de la RBC y de la red de la profesora

\begin{tabular}{|c|c|c|c|}
\hline & \begin{tabular}{|l|} 
Conceptos Nucleares \\
RAP-1
\end{tabular} & $\begin{array}{l}\text { Conceptos Nucleares } \\
\text { RAP-2 }\end{array}$ & $\begin{array}{l}\text { Conceptos Nucleares } \\
\text { RAP-3 }\end{array}$ \\
\hline Grupo 1B & 1-Medir 2-Unidad & 1-Medir 2-SMD & $\begin{array}{ll}\text { 1-Medir } & \text { 2-Múltiplos y } \\
& \text { submúltiplos de } 10\end{array}$ \\
\hline Grupo 1C & $\begin{array}{l}\text { 1-Unidad } \\
\text { 2-Instrumento de } \\
\text { medida }\end{array}$ & $\begin{array}{l}\text { 1-Unidad } \\
2 \text {-Instrumento de } \\
\text { medida }\end{array}$ & 1-Medir \\
\hline RBC & $\begin{array}{l}\text { 1-SMD 2-Unidad } \\
\text { 3-Magnitud }\end{array}$ & & \\
\hline Red de la profesora & 1-Unidad 2-Medir & & \\
\hline
\end{tabular}


Las conclusiones obtenidas a partir de estos datos son similares a las extraídas de los índices de complejidad, y es que las estructuras cognitivas de los estudiantes tienden a mantenerse estables en tiempo o bien a ser más simples coincidiendo con Casas y Luengo (2004).

Fijándonos en la tabla 34, si comparamos los Conceptos Nucleares de los estudiantes con los existentes en la RBC y en la Red de la profesora, observamos que los estudiantes tienen el mismo número de Conceptos Nucleares en su estructura cognitiva que la profesora y uno menos que la RBC, lo cual apoya los resultados obtenidos sobre la complejidad de las redes visto anteriormente.

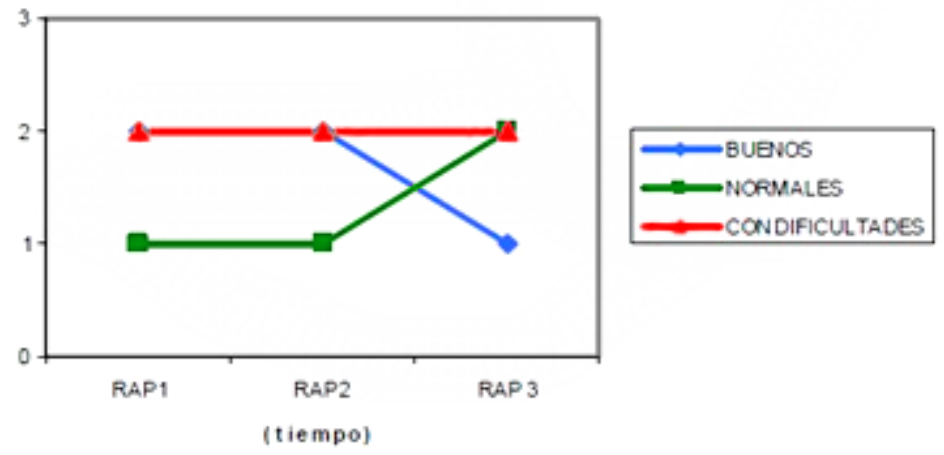

Figura 13. Conceptos nucleares para grupos en función de la calificación

Tabla 7. Clasificación de los Conceptos Nucleares de las Redes Medias de alumnos según su calificación

\begin{tabular}{|c|c|c|c|}
\hline & $\begin{array}{c}\text { CONCEPTOS } \\
\text { NUCLEARES } \\
\text { RAP1 }\end{array}$ & $\begin{array}{c}\text { CONCEPTOS } \\
\text { NUCLEARES } \\
\text { RAP2 }\end{array}$ & $\begin{array}{c}\text { CONCEPTOS } \\
\text { NUCLEARES } \\
\text { RAP 3 }\end{array}$ \\
\hline BUENOS & 2 & 2 & 1 \\
NORMALES & 1 & 1 & 2 \\
CON DIFICULTADES & 2 & 2 & 2 \\
\hline
\end{tabular}

Analizando la figura 13 y la tabla 7 , podemos constatar que las estructuras cognitivas de los alumnos que "presentan dificultades" permanecen estables en el tiempo, mientras que las de los alumnos con mejores notas suelen hacerse más sencillas. Estos resultados concuerdan con los que hemos obtenido en el Índice de Coherencia de Redes. 
Similaridad de las redes medias con respecto a la RBC y a la red de la profesora

En este apartado, analizaremos cómo de similares son las redes de los alumnos, por una parte, comparándola con la RBC y por otra, con la red de la profesora.

En términos generales, y cómo podemos observar en las tablas 8 y 9 , las redes de los alumnos se encuentran alejadas, tanto de la Red Básica Conceptual, como de la red de la profesora, aunque se encuentran algo más próximas a esta última.

Tabla 8. Índice de similaridad, con la RBC, de las Redes Medias de alumnos según su curso

\begin{tabular}{|c|c|c|c|}
\hline & Similaridad & $\begin{array}{c}\text { Similaridad RAP-2 } \\
\text { con RBC }\end{array}$ & $\begin{array}{c}\text { Similaridad RAP-3 } \\
\text { con RBC }\end{array}$ \\
\hline Grupo1B 12 ESO & 0,25 & 0,15384615 & 0,25 \\
Grupo1C 12 ESO & 0,07142857 & 0,07142857 & 0,07142857 \\
\hline
\end{tabular}

Tabla 9. Índice de similaridad, con la red de la profesora, de las Redes Medias de alumnos según su curso

\begin{tabular}{|c|c|c|c|}
\hline & $\begin{array}{c}\text { Similaridad RAP-1 } \\
\text { con la red } \\
\text { de la profesora }\end{array}$ & $\begin{array}{c}\text { Similaridad RAP-2 } \\
\text { con la red } \\
\text { de la profesora }\end{array}$ & $\begin{array}{c}\text { Similaridad RAP-3 } \\
\text { con la red } \\
\text { de la profesora }\end{array}$ \\
\hline Grupo1B 12 ESO & 0,27272727 & 0,27272727 & 0,27272727 \\
Grupo1C 12 ESO & 0,4 & 0,4 & 0,27272727 \\
\hline
\end{tabular}

Si nos centramos en analizar los datos obtenidos de la similaridad en función de la calificación, podemos observar las siguientes figuras:

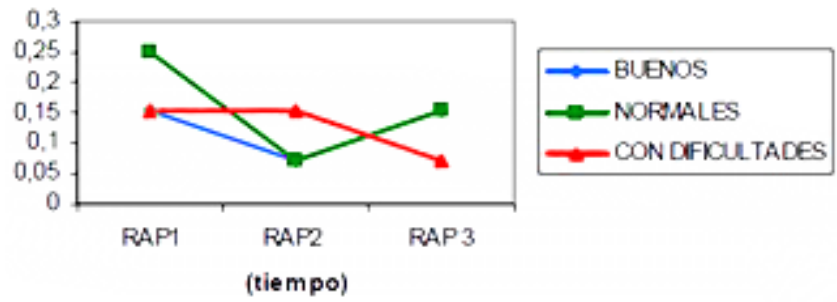

Figura 14. Similaridad con la RBC de grupos en función de la calificación 


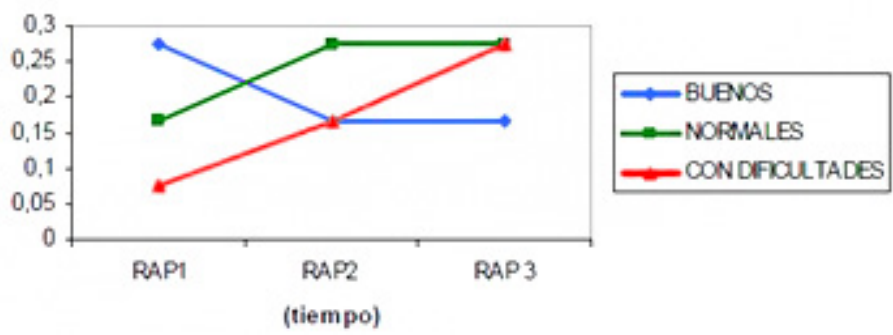

Figura 15. Similaridad con la red de la profesora de grupos en función de la calificación

Se puede decir que, al igual que ocurría con los datos en función del grupo al que pertenecen los estudiantes, las redes de estos siguen estando alejadas tanto de la $\mathrm{RBC}$ como de la red de la profesora.

\section{Conclusiones}

Tratando de cumplir con el propósito general de esta investigación, se ha realizado un estudio en el que se analizan las estructuras o redes cognitivas de los alumnos, así como su estabilidad a lo largo del tiempo. Además, hemos profundizado en una nueva forma de evaluación del aprendizaje, a través del análisis de las RAP al abordar una nueva Unidad Didáctica.

Las conclusiones que se han obtenido de este estudio son las siguientes:

1. Observando la estructura cognitiva que presenta un estudiante de $1^{\circ} \mathrm{ESO}$, en relación al tema del SMD, podemos concluir que es una estructura menos compleja que la que presenta la RBC y que la de la profesora, encontrando bastantes diferencias entre ellas.

2. Las redes de los alumnos, en términos generales, se encuentran alejadas tanto de la Red de la profesora, como de la RBC.

3. Podemos decir que las estructuras que presentan una mayor coherencia son las de los alumnos "buenos", y todas, en general pueden permanecer estables o hacerse más sencillas a lo largo del tiempo, coincidiendo con lo que Casas y Luengo (2004) defienden en sus resultados de la TCN.

4. Hemos podido observar que las redes de los alumnos "buenos" no tienen por qué ser más similares a la RBC que las del resto, de hecho, estos alumnos presentan unas redes más alejadas tras la práctica con la Unidad Didáctica.

5. Hablando del "poso cultural" y de la estabilidad de las redes cognitivas de los alumnos, podemos deducir que los alumnos "buenos", tanto su complejidad, como el número de conceptos nucleares se reduce a lo largo de la investigación. Por otro lado, en los alumnos que "presentan dificultades" se mantienen más estables. Ambos resultados coinciden y apoyan la TCN de Casas y Luengo (2004). 


\section{Líneas de investigación abiertas}

Teniendo en cuenta las conclusiones de nuestro estudio, quedan abiertas las líneas siguientes:

Diseños mixtos Cualitativo-Cuantitativos:

- Realizar entrevistas semiestructuradas que nos permitan analizar mediante softwares cualitativos (como WebQDA) los datos, para poder explicar los significados entre las uniones de nodos.

- Utilizar un enfoque cualitativo que nos permita analizar las redes de los alumnos de una forma más detallada.

Profundizar en la aplicación de la TCN:

- Comprobar la variación en los resultados al utilizar UD diseñadas en base a TCN y evaluadas con diferentes métodos.

- Investigar sobre la elaboración y construcción de la RBC ya que no existen suficientes investigaciones que determinen con certeza sus criterios de construcción y significancia.

Profundizar en el empleo de las RAP:

- Analizar todas las posibilidades que las RAP nos aporta, como posible herramienta que nos permita evaluar a los alumnos.

- Detección de los tipos de aprendizaje mediante el análisis de la variación de las RAP.

\section{Referencias}

Arias, J. (2008). Evaluación de la calidad de Cursos Virtuales- Indicadores de Calidad y construcción de un cuestionario de medida. Aplicación al ámbito de asignaturas de Ingeniería Telemática (Tesis Doctoral). Universidad de Extremadura, Badajoz.

Ausubel, D., Novak, J. y Hanesian, H. (1978). Psicología educativa: un punto de vista cognoscitivo. México: Ed. Trillas.

Bizarro, N., Luengo, R., Casas, L. M. y Torres, J. L. (2015). Aplicación de las Redes Asociativas Pathfinder al análisis de los conceptos forma, tamaño y color en alumnos con Discapacidad Intelectual. Revista Lusófona de Educação, 29(29), 121-144.

Carvalho, J.L. (2011). Estudio de las posibilidades de aplicación a la enseñanza de la Matemática del entorno PmatE: Validación y aportaciones en $1^{\circ}$ Ciclo de Enseñanza Básica de Portugal (Tesis Doctoral). Universidad de Extremadura, Badajoz.

Casas, L. (2002). El estudio de la estructura cognitiva de alumnos a través de Redes Asociativas Pathfinder. Aplicaciones y posibilidades en Geometría (Tesis Doctoral). Universidad de Extremadura, Badajoz. 
Contreras, J. Á., Arias, J., Luengo, R., y Casas, L. M. (2016). Método complementario de obtención de los Conceptos Nucleares para la Teoría de los Conceptos Nucleares. El Índice de Nuclearidad. IE Comunicaciones: Revista Iberoamericana de Informática Educativa, 23, 35-50.

Casas, L. y Luengo, R. (2004). Representación del conocimiento y aprendizaje. Teoría de los conceptos nucleares. Revista Española de Pedagogía, 62(227), 59-84.

Godinho, V., Luengo, R. y Casas, L. (2011). Software MICROGOLUCA: Knowledge Representation in Mental Calculation. US-China Education Review, 1 (4), 592-600.

Hidalgo, V. (2007). Aproximación a la medida del aprendizaje mediante redes asociativas Pathfinder. (Trabajo fin de Máster). Universidad de Extremadura, Badajoz.

Luengo R. (2013). La Teoría de los Conceptos Nucleares y su aplicación en la investigación en didáctica de las matemáticas. (Firma Invitada). Revista: UNION. Rev. Iberoamericana de educación Matemática, 34, 9-36.

Picado, M. Rico, L. y Gómez, B. (2013). El Sistema Métrico Decimal en un libro de texto de matemáticas para la instrucción primaria en las Islas Canarias en el s. XIX. NÚMEROS. Revista de Didáctica de las Matemáticas, 82, 37-53.

Roldán, I. (2013). Estabilidad de redes cognitivas en el aprendizaje del Sistema Métrico Decimal en alumnos de $1^{\circ} \mathrm{ESO}$ (Trabajo Final de Master). Universidad de Extremadura, Badajoz.

Roldan, I., Soto, L. M., Luengo, R. y Carvalho, J. L. (2017). Evolución de las redes cognitivas en el aprendizaje del Sistema Métrico Decimal utilizando Redes Asociativas Pathfinder. En Costa, A., Sánchez-Gómez, M., y Martín, M. (Eds.), Actas del $7^{\circ}$ Congreso Ibero-Americano en Investigación Cualitativa Nolumen 1 - Investigación Cualitativa en Educación) (pp. 36-45). Salamanca - ESPAÑA: Ludomedia.

Schvaneveldt, R. (1989). Pathfinder Associative Networks. Studies in Knowledge Organization. Norwood: Ablex.

Veríssimo, S., Casas, L. y Luengo, R. (2013). La introducción de las ideas de la Teoría de los Conceptos Nucleares en la enseñanza de la geometría y sus implicaciones (Tesis Doctoral). Badajoz: Universidad de Extremadura.

Isabel Maria Roldán

Grupo de Investigación: CIBERDIDACT

Facultad de Educación, Universidad de Extremadura, Badajoz Departamento de Didáctica de las Ciencias Experimentales y Matemáticas

Email: isabelroldan@gmail.com

Luis Manuel Soto Ardila

Grupo de Investigación: CIBERDIDACT

Facultad de Educación, Universidad de Extremadura, Badajoz Departamento de Didáctica de las Ciencias Experimentales y Matemáticas

Email: luismanuel@unex.es ORCID: 0000-0002-1041-4077 
Ricardo Luengo González Grupo de Investigación: CIBERDIDACT

Facultad de Educación, Universidad de Extremadura, Badajoz Departamento de Didáctica de las Ciencias Experimentales y Matemáticas

Email: rluengo@unex.es

ORCID: 0000-0003-4349-8606

José Luís Torres Carvalho

Grupo de Investigación: CIBERDIDACT

Facultad de Educación, Universidad de Extremadura, Badajoz Departamento de Didáctica de las Ciencias Experimentales y Matemáticas

Email: jltc@unex.es ORCID: 0000-0001-6384-7821

Correspondência

Grupo de Investigación CiberDidact Facultad de Educación - Universidad de Extremadura Avda. de Elvas S/N; 06006 Badajoz; España

Data de submissão: Fevereiro de 2017

Data de avaliação: Abril de 2017

Data de publicação: Julho 2018 